\title{
Sexually risky behaviour in adolescents was reduced by a safer sex, condom based intervention
}

\author{
Jemmott JB 3rd, Jemmott LS, Fong GT. Abstinence and safer sex HIV risk-reduction interventions for African American adolescents. \\ A randomized controlled trial.JAMA 1998 May 20;279:1529-36.
}

\section{Question}

Will a multicomponent abstinence intervention reduce the frequency of sexual intercourse, and will a safer sex intervention increase condom use in African-American adolescents?

\section{Design}

Randomised controlled trial (RCT) with 12 months follow up.

\section{Setting}

3 middle schools in low income African-American communities in Philadelphia, Pennsylvania, USA.

\section{Participants}

School announcements and letters to parents and guardians were used to recruit students. 659 adolescents in the 6 th and 7 th grade were studied (mean age 11.8 y, 53\% girls, 26.8\% living with both parents, $25 \%$ reported previous sexual experience).

\section{Intervention}

Adolescents were paid US $\$ 100$ to participate. All interventions were given in 8 one hour sessions on 2 consecutive Saturdays. They were designed to be educational, entertaining, and culture sensitive and included group discussion, videos, games, brainstorming, and experiential and skill building exercises to encourage pride in self and community, responsible behaviour, and consideration of goals and long term health concerns. 220 adolescents were allocated to an abstinence intervention with goals of increasing knowledge of HIV and other sexually transmitted diseases, strengthening behavioural beliefs supporting abstinence, and increasing self efficacy and skills that promote abstinence. 221 adolescents were allocated to a safer sex intervention with goals centred on effective condom use, allaying fears about reduced sexual enjoyment, and increasing skills and self efficacy in their use. 218 control adolescents were allocated to a similar intervention that focused on healthy lifestyle (diet, exercise, and smoking). Groups were led by an adult facilitator or peer cofacilitators.

\section{Main outcome measures}

Sexual behaviours (frequency of intercourse and condom use).

\section{Main results}

Adolescents in the abstinence group reported less sexual intercourse at 3 months $(12.5 \%$ v $16.6 \%$ for safer sex group and $21.5 \%$ for the control group, $\mathrm{p}=0.02$ for abstinence $v$ other groups), but not at 6 and 12 months. Adolescents in the safer sex group reported a higher mean frequency of condom use than the control group at 3, 6, and 12 months. For adolescents who were sexually experienced at baseline, those in the safer sex group had lower rates of sexual intercourse at 6 and 12 months. They also had a lower adjusted mean frequency of unprotected sexual intercourse at 3 months ( $\mathrm{p} \leqslant 0.002$ for safer sex $v$ others), 6 months $(\mathrm{p}=0.03$ for safer sex $v$ control), and 12 months $(\mathrm{p}<0.001$ for safer sex $v$ others $)$.

\section{Conclusion}

Safer sex intervention increased condom use and for those who reported sexual experience at baseline, reduced rates of intercourse.

Source of funding: National Institute of Mental Health.

For correspondence:Dr J B Jemmott 3rd, Department of Psychology, Green Hall, Princeton University, Princeton, NJ 08544-1010, USA. Fax +1 6092581275.

\section{Commentary}

The study by Jemmott $e t$ al represents the first RCT to evaluate the effectiveness of a condom based intervention compared with an abstinence based intervention and an attention control group. Kirby et al compared an abstinence programme with a no intervention control group and found that adolescents in both groups were equally likely to become sexually active or report pregnancy or a sexually transmitted infection. ${ }^{1}$ Jemmott $e t$ al did not find a long term impact of the abstinence programme but did find that the safer sex intervention increased condom use and, for those sexually experienced at baseline, reduced rates of sexual intercourse which were sustained. This is contrary to the commonly held belief that sex education encourages sexual activity.

This study had a large sample size, an attention control group that reduced the potential confounding of study results (Hawthorne effect), data collectors blinded to the intervention, and an excellent follow up rate. Lower income African-American adolescents, few of whom lived with both parents, were studied. These findings may not be generalisable to adolescents in different circumstances. Data collection relied on self reporting of sensitive information; however, participants signed an agreement pledging to answer the questions honestly.

What are the implications for nurses? Firstly, a community based, safer sex intervention for adolescents can be mounted; secondly, if the goal is to reduce unprotected sexual intercourse, the safer sex strategy is more effective than an abstinence intervention, especially over the longer term; thirdly, although peer facilitators were evaluated more favourably, this did not improve outcomes and appropriately trained adults or peers therefore can be used; fourthly, the intervention should be intensive, theory based, and culture sensitive; fifthly, attendance at the intervention might be enhanced by incentives consistent with the tastes and desires of adolescents. The challenge still remains to identify and test programmes that ensure a long term effect.

$$
\begin{array}{r}
\text { Willa M Doswell, RN, PhD } \\
\text { Assistant Professor, School of Nursing } \\
\text { University of Pittsburgh } \\
\text { Pittsburgh, Pennsylvania, USA }
\end{array}
$$

1 Kirby D, Korpi M, Barth RP, et al. The impact of postponing sexual involvement curriculum among youths in California. Fam Plann Perspect 1997;29:100-8. 